\title{
MSCs promote fistula closure in Crohn's disease
}

Results from a new study show that treatment with allogeneic mesenchymal stem cells (MSCs) leads to a greater remission rate than placebo in patients with treatment-refractory perianal fistulas associated with Crohn's disease.

Perianal fistulas are a commonly occurring complication in patients with Crohn's disease, causing substantial morbidity and markedly reducing quality of life. New treatments are desperately needed: these fistulas are frequently refractory to conventional therapy, such as anti-TNF agents, and often require surgical intervention. As epithelial defects related to chronic inflammation are thought to be involved in their pathogenesis, MSCs have been advanced as a potential treatment, due to their anti-inflammatory and regenerative properties.

"Various research groups have undertaken initiatives to determine the potential for MSC-based therapy in a variety of immune-mediated inflammatory disorders,"

says author Julián Panés. "Previous phase I studies using local injection of autologous MSCs of adipose or bone marrow origin showed closure of perianal fistulas in $60-85 \%$ of patients with Crohn's disease."

To evaluate the efficacy of MSCs for treating complex perianal fistulas, the researchers conducted a phase III, randomized, double-blind placebo-controlled study across eight countries. Given that autologous MSCs are expensive and difficult to generate quickly and in large numbers, allogeneic MSCs derived from healthy donor adipose tissue were used. 212 adults with Crohn's disease and treatment-refractory perianal fistulas were randomly assigned to local MSC injection or placebo; the combined primary end point (clinical closure of fistulas and absence of abscess $>2 \mathrm{~cm}$ as demonstrated by MRI) was assessed at 24 weeks.

A greater number of patients receiving MSCs achieved the primary end point than those receiving placebo (50\% versus $36 \%$, respectively; $P=0.024)$. Notably, MSC treatment was well-tolerated and safe, with no evidence of immunoreactivity.

"For patients that failed immunosuppressants and TNF antagonists, therapy with MSCs would be the only alternative to surgery," concludes Panés.

Hugh Thomas

ORIGINAL ARTICLE Panés, J. et al. Expanded allogeneic adipose-derived mesenchymal stem cells (Cx601) for complex perianal fistulas in Crohn's disease: a phase 3 randomised, doubleblind controlled trial. Lancet http://dx.doi.org/ 10.1016/S0140-6736(16)31203-X (2016) 